Pesq. Vet. Bras. 36(2):136-140, fevereiro 2016

DOI: $10.1590 /$ S0100-736X2016000200012

\title{
Miologia do membro torácico da paca (Cuniculus paca) ${ }^{1}$
}

\author{
Leonardo M. Leal ${ }^{2 *}$, Juliana A.C.E. Silva ${ }^{3}$, Fabricio S. Oliveira ${ }^{3}$, Tais H.C. Sasahara ${ }^{3}$ \\ e Marcia R.F. Machado ${ }^{3}$
}

\begin{abstract}
Leal L.M., Silva J.A.C.E., Oliveira F.S., Sasahara T.H. \& Machado M.R.F. 2016. [Miology of the forelimb of paca (Cuniculus paca).] Miologia do membro torácico da paca (Cuniculus paca). Pesquisa Veterinária Brasileira 36(2):136-140. Departamento de Morfologia e Fisiologia Animal, Faculdade de Ciências Agrárias e Veterinárias, Universidade Estadual Paulista, Via de Acesso Prof. Paulo Donato Castellane s/n, Jaboticabal, SP 14884-900, Brazil. E-mail: morfologia@fcav.unesp.br

The objective was to describe the forelimb muscles of paca (Cuniculus paca Linnaeus, 1766), by anatomical dissection of this region. Five adult male and female $C$. paca, weighing $5-10 \mathrm{~kg}$, from the Department of Wild Animals, FCAV-Unesp, Jaboticabal/SP, were used. The animals were fixed in formaldehyde $10 \%$ and stored in saline $30 \%$ for anatomic dissection of the muscles of shoulder, arm and forearm, identifying the origin and insertion of each muscle. The results were photodocumented and discussed based on the literature of domestic animals, rats and guinea pigs. Unless some variances in the origin and insertion of each muscle and fusion of bellies of some muscle groups, in general, the muscles of shoulder, arm and forearm of paca resemble the ones of domestic animals and other rodents.
\end{abstract}

INDEX TERMS: Muscles, paca, Cuniculus paca, rodent, wild.

RESUMO.- Objetivou-se descrever os músculos do membro torácico da paca (Cuniculus paca Linnaeus, 1766), mediante dissecação anatômica dessa região. Foram utilizadas cinco Cuniculus paca adultas, machos e fêmeas, pesando entre cinco e $10 \mathrm{~kg}$ do plantel de pacas do setor de Animais Silvestres da FCAV, Unesp, Jaboticabal/SP. Os animais foram fixados em formoldeído a 10\% e conservados em solução salina a 30\% para dissecação anatômica da musculatura do ombro, braço e antebraço, identificando-se a origem e inserção de cada músculo. Os resultados foram fotodocumentados e discutidos com base na literatura sobre animais domésticos, ratos e cobaias. Salvo algumas variâncias na origem e inserção de cada músculo e na fusão dos ventres de alguns grupos musculares, de forma geral, a musculatura do ombro, braço e antebraço da paca assemelha-se a dos animais domésticos e a de outros roedores.

\footnotetext{
${ }^{1}$ Recebido em 3 de maio de 2015.

Aceito para publicação em 15 de janeiro de 2016.

${ }^{2}$ Departamento de Medicina Veterinária, Faculdade Ingá, Unidade de Ensino Superior Ingá (Uningá), Rodovia PR-317 no.6114, Parque Industrial Duzentos, Maringá, PR 87035-510, Brasil. *Autor para correspondência: leonardo.vet@hotmail.com

${ }^{3}$ Departamento de Morfologia e Fisiologia Animal, Faculdade de Ciências Agrárias e Veterinárias (FCAV), Universidade Estadual Paulista (Unesp), Via de Acesso Prof. Paulo Donato Castellane s/n, Jaboticabal, SP 14884900, Brasil.
}

TERMOS DE INDEXAÇÃO: Músculos, paca, Cuniculus paca, roedor, selvagem.

\section{INTRODUÇÃO}

A paca (Cuniculus paca Linnaeus, 1766) é uma espécie pertencente à ordem dos roedores, típica de regiões tropicais (Redford \& Robinson 1991), presente em grande parte do território brasileiro e América latina (Eisenberg \& Redford 1999, Lange \& Schmidt 2007); alimenta-se de frutas, mas pode consumir outros vegetais e até insetos em períodos de escassez alimentar (Dubost \& Henry 2006). Pode atingir $80 \mathrm{~cm}$ de comprimento, $12 \mathrm{~kg}$ de massa corpórea (Pachaly et al. 2001) e 16 anos de tempo médio de vida (Eisenberg \& Redford 1999, Lange \& Schmidt 2007).

O conhecimento morfológico é fundamental para a conservação de espécies selvagens (Santos et al. 2010a, 2010b, Vaz et al. 2011, Rosa et al. 2012). Descrições anatômicas contribuem para compor o acervo teórico sobre as espécies e para o enriquecimento das aplicações na clínica e cirurgia veterinária (Aversi-Ferreira et al. 2005, Vavruk 2012).

De outra forma, o mercado consumidor de carne tem se mostrado bastante receptivo ao consumo de carnes exóticas (Bressan et al. 2004). Gomes et al. (2013), afirmam que a carne da paca se apresenta sensorialmente semelhante à carne suína e com boa aceitação pelos consumidores. A maior parte dos cortes de carne comumente encontrados 
na indústria alimentícia é baseada nas divisões anatômicas dos grupos musculares (Mucciolo \& Paiva 1943, Almeida Junior et al. 2004). Deste modo, o conhecimento anatômico muscular da espécie é imprescindível para a determinação de seus cortes comerciais.

Assim, diante da escassez de estudos na literatura sobre a miologia deste roedor e considerando-se a necessidade do conhecimento anatômico detalhado desta espécie para sua conservação e importância zootécnica, objetiva-se identificar e descrever a origem e inserção de cada músculo do ombro, braço e antebraço da paca.

\section{MATERIAL E MÉTODOS}

A presente metodologia foi aprovada pelo Comitê de Ética no Uso de Animais (CEUA) da Faculdade de Ciências Agrárias e Veterinárias (FCAV-Unesp), Jaboticabal-SP, de acordo com o Colégio Brasileiro de Experimentação Animal (COBEA) sob o número de protocolo 017754/13.

Foram utilizados cinco Cuniculus paca adultas, machos e fêmeas, pesando entre cinco e $10 \mathrm{~kg}$ do plantel de pacas do setor de Animais Silvestres da FCAV, Unesp, Jaboticabal-SP, que é registrado no Instituto Brasileiro do Meio Ambiente e dos Recursos Naturais Renováveis - IBAMA, como criatório de espécimes da fauna brasileira para fins científicos (cadastro de registro - 482508).

A eutanásia foi realizada mediante sedação prévia com meperidina $(3 \mathrm{mg} / \mathrm{kg})$ associada a midazolan $(1 \mathrm{mg} / \mathrm{kg})$ pela via intramuscular, anestesia geral com quetamina $(25 \mathrm{mg} / \mathrm{kg})$ e xilazina $(0,5 \mathrm{mg} / \mathrm{kg})$ também pela via intramuscular, e por fim injeção intracardíaca de cloreto de potássio 19,1\%, dose-efeito, até a parada cardiorespiratória.

Logo após a eutanásia, no Laboratório de Anatomia da FCAV, Unesp-Jaboticabal, SP, os animais foram fixados em solução de formaldeído $10 \%$ e conservados em solução salina a 30\% (Oliveira 2014) para a posterior dissecação.

Os membros torácicos, direito e esquerdo, foram dissecados e os músculos do ombro, braço e antebraço foram identificados, bem como suas origens e inserções. Os resultados foram fotodocumentados e discutidos com base na literatura disponível sobre o rato (Mus norvegicus albinus), a cobaia (Cavia porcellus) e os animais domésticos. A nomenclatura anatômica adotada baseou-se na Nomenclatura Anatômica Veterinária Ilustrada (Schaller 1999).

\section{RESULTADOS}

Após a retirada da pele e músculo cutâneo, verificou-se na conformação do ombro e braço de Cuniculus paca, os seguintes músculos: músculo (m.) trapézio, cervical e torácico; m. omotransverso; m. romboide; m. infraespinhoso; m. supraespinhoso; m. deltoide, escapular e acromial; m. redondo menor; m. redondo maior; m. subescapular; m. peitoral, superficial e profundo; $\mathrm{m}$. serrátil; $\mathrm{m}$. grande dorsal; $\mathrm{m}$. braquicefálico; m. braquial; m. coracobraquial; m. bíceps braquial; m. tríceps e m. tensor da fáscia do antebraço (Fig.1 e 2).

De forma geral a paca apresenta um grande volume muscular recobrindo os ossos do membro torácico e a união dos ventres dos distintos grupos musculares é comum. Constatou-se em todos os animais que o $\mathrm{m}$. trapézio cervical, m. deltoide escapular e m. braquicefálico se unem, dificultando a delimitação exata de cada grupo muscular. 0 mesmo ocorre com os músculos supraespinhoso e subescapular que se unem na borda cranial da escápula. Os músculos supraespinhoso e infraespinhoso também são aderidos,

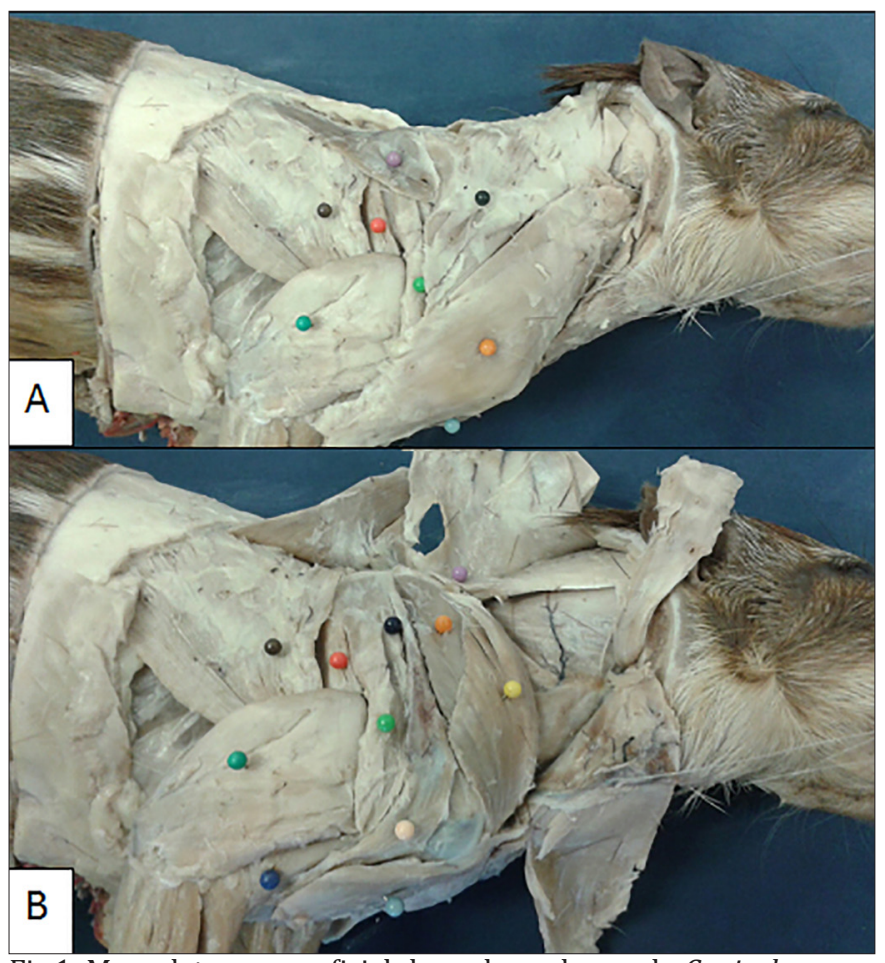

Fig.1. Musculatura superficial do ombro e braço de Cuniculus paca adulta. A pele e a musculatura cutânea foram removidas. (A) Vista lateral, observam-se m. trapézio cervical (bola preta), $\mathrm{m}$. trapézio torácico (bola lilás), m. grande dorsal (bola marrom), $\mathrm{m}$. redondo maior (bola vermelha), m. deltoide escapular (bola verde clara), m. braquicefálico (bola laranja), m. tríceps (bola verde escura) e m. peitoral superficial (bola azul clara). (B) Vista lateral com mm. trapézios e braquicefálico rebatidos, visibiliza-se: m. romboide (bola lilás), m. grande dorsal (bola marrom), m. redondo maior (bola vermelha), m. deltoide escapular (bola verde clara), m. deltoide acromial (bola salmão), m. infraespinhoso (bola preta), m. supraespinhoso (bola laranja), m. omotransverso (bola amarela), m. tríceps (bola verde escura), m. braquial (bola azul escura) e m. peitoral superficial (bola azul clara).

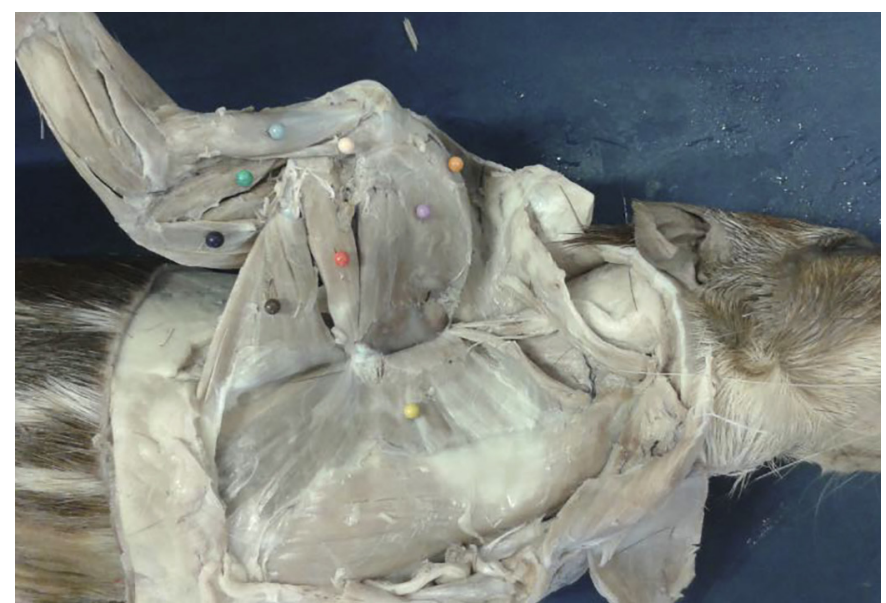

Fig.2. Musculatura do ombro e braço de Cuniculus paca adulta em vista medial. A pele, a musculatura cutânea e os músculos peitorais e braquicefálico foram removidos. Observam-se $\mathrm{m}$. serrátil (bola amarela), m. grande dorsal (bola marrom), m. redondo maior (bola vermelha), m. subescapular (bola lilás), $\mathrm{m}$. supraespinhoso (bola laranja), $\mathrm{m}$. tensor da fáscia do antebraço (bola preta), m. tríceps (bola verde escura), m. bíceps braquial (bola azul clara) e m. coracobraquial (bola salmão). 


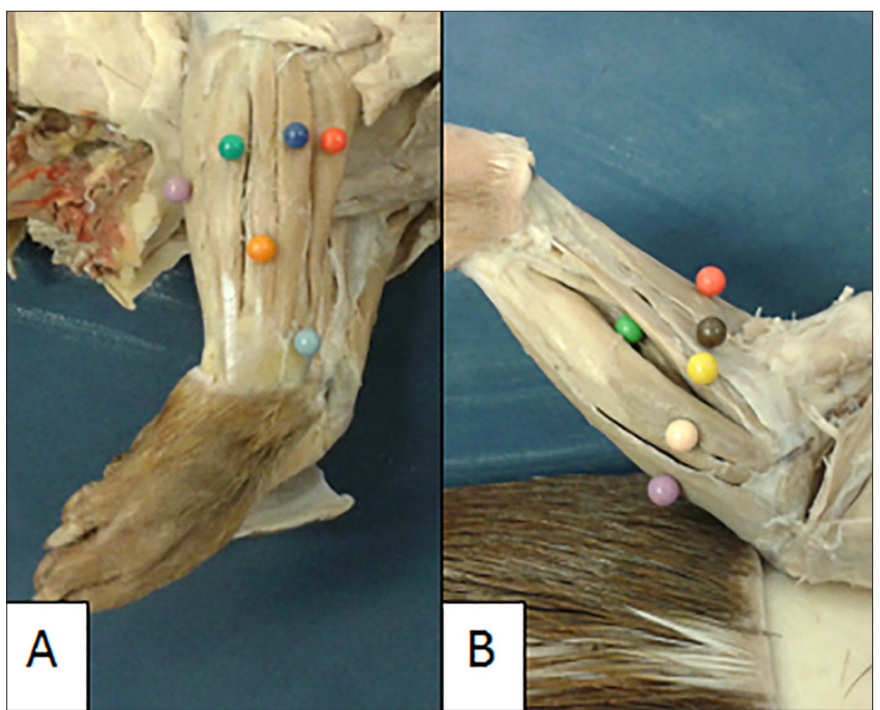

Fig.3. Musculatura do antebraço de Cuniculus paca adulta. A pele e a musculatura cutânea foram removidas. (A) Vista lateral, observam-se m. flexor ulnar do carpo (bola lilás), m. ulnar lateral (bola verde escura), m. extensor digital lateral (bola laranja), m. extensor comum dos dedos (bola azul escura), m. extensor oblíquo do carpo (bola azul clara) e m. extensor radial do carpo (bola vermelha). (B) Vista medial, visibilizam-se m. flexor ulnar do carpo (bola lilás), m. flexor superficial dos dedos (bola salmão), m. flexor profundo dos dedos (bola verde clara), m. flexor radial do carpo (bola amarela), m. pronador redondo (bola marrom) e m. extensor radial do carpo (bola vermelha). porém a espinha da escapula auxilia na sua identificação e isolamento de cada músculo.

Na paca, o músculo omotransverso não é superficial, fica recoberto pelos músculos braquicefálico e trapézio.

Verificou-se os seguintes músculos do antebraço da paca: m. ancôneo; m. extensor radial do carpo; m. supinador; m. extensor obliquo do carpo; m. extensor comum dos dedos; m. extensor lateral dos dedos; m. ulnar lateral; m. flexor ulnar do carpo; m. flexor superficial dos dedos; m. flexor digital profundo; $\mathrm{m}$. flexor radial do carpo e m. pronador redondo (Fig.3).

A origem e a inserção de cada músculo do membro torácico da paca foram identificadas e estão listadas em ordem alfabética no Quadro 1.

\section{DISCUSSÃO}

De forma geral, os músculos que compõem o ombro e braço de Cuniculus paca, salvo algumas especificidades, são os mesmo descritos para animais domésticos e roedores (Greene 1955, Cooper \& Schiller 1975, Sisson \& Grossman 1986, Schaller 1999, Dyce et al. 2010, König \& Liebich 2011).

Apesar da anatomia da paca ser semelhante aos animais domésticos quanto aos músculos identificados no membro torácico, neste roedor é comum a união dos ventres musculares, dificultando o isolamento de cada músculo. Na paca, o m. trapézio cervical, m. deltoide escapular e m. braquicefálico se unem na região craniolateral superficial da

\section{Quadro 1. Listagem, em ordem alfabética, da origem e inserção dos músculos do membro torácico da paca} (Cuniculus paca). Jaboticabal/SP, 2015

\begin{tabular}{|c|c|c|}
\hline Músculo & Origem & Inserção \\
\hline Ancôneo & Epicôndilo medial do úmero & Olécrano \\
\hline Bíceps braquial & Tubérculo supraglenoide da escápula & $\begin{array}{l}\text { Tuberosidade medial da extremidade proximal } \\
\text { do radio e parte adjacente a ulna }\end{array}$ \\
\hline Braquiocefálico & Occipital & $\begin{array}{l}\text { Espinha da escápula e fáscia muscular, na face } \\
\text { cranial da articulação do cotovelo. }\end{array}$ \\
\hline Braquial & Face caudolateral proximal do tubérculo maior do úmero & Cabeça do rádio \\
\hline Coracobraquial & Cabeça do úmero & Diáfise medial do úmero \\
\hline Deltoide escapular & Espinha da escapula borda caudal & Tuberosidade deltoide \\
\hline Extensor comum dos dedos & Epicôndilo lateral do úmero & Falange distal dos dedos II-IV \\
\hline Extensor lateral dos dedos & Epicôndilo lateral do úmero & Falange proximal dos dedos III-V \\
\hline Extensor oblíquo do carpo & Região craniolateral distal do rádio & Falange proximal do dedo I \\
\hline Extensor radial do carpo & Epicôndilo lateral do úmero & Metacárpico II e III \\
\hline Flexor profundo dos dedos & Epicôndilo medial do úmero, rádio e ulna & Falange distal dos dedos \\
\hline Flexor superficial dos dedos & Epicôndilo medial do úmero & Extremidade proximal na falange media \\
\hline Flexor radial do carpo & Epicôndilo medial do úmero & Extremidade proximal do metacarpo II \\
\hline Grande dorsal & Fáscia tóracolombar & Tuberosidade redonda maior do úmero \\
\hline Infraespinhoso & Fossa supraespinhal da escápula & Tubérculo maior lateral do úmero \\
\hline Omotransverso & Espinha da escápula na fáscia com supraespinhoso & Clavícula \\
\hline Peitoral Profundo & Esterno & Face medial do tubérculo maior do úmero \\
\hline Peitoral Superficial & Esterno & Diáfise craniolateral do úmero \\
\hline Pronador redondo & Epicôndilo medial do úmero & Face medial do rádio \\
\hline Redondo Maior & Borda caudodorsal da escápula & Tuberosidade redonda maior \\
\hline Redondo Menor & Borda caudoventral da escápula & Tubérculo maior do úmero \\
\hline Romboide & Rafe mediana do pescoço & Superfície dorsal da escápula \\
\hline Serrátil & Processos transversos das vértebras cervicais & Face serrátil da escápula \\
\hline Subescapular & Face subescapular da escápula & Tubérculo menor \\
\hline Supinador & Epicôndilo lateral do úmero & Região proximal do rádio \\
\hline Supraespinhoso & Fossa supraespinhal da escápula & Tubérculo maior do úmero \\
\hline Tensor da fáscia do antebraço & Fáscia do tendão do grande dorsal & Olécrano \\
\hline Trapézio - porção torácica & Rafe mediana cervical caudal & Região caudodorsal da espinha da escápula \\
\hline Trapézio -porção cervical & Rafe mediana cervical cranial & Espinha da escápula \\
\hline
\end{tabular}


escápula, semelhantemente as cobaias (Cooper \& Schiller 1975). Em equinos, o processo ocorre semelhante para o músculo deltoide, pois, se funde parcialmente ao músculo infraespinhoso (König \& Liebich 2011). 0 mesmo ocorre com os músculos supraespinhoso e subescapular que se unem na borda cranial profunda da escápula, também em equinos. Na paca, os músculos supraespinhoso e infraespinhoso também são aderidos, porém a espinha da escapula auxilia na sua identificação e isolamento de cada músculo.

$\mathrm{Na}$ paca, o músculo trapézio é divido em duas partes, cervical e torácica, assim como na cobaia, no rato e na maioria dos animais domésticos (Cooper \& Schiller 1975, Greene 1955, Sisson \& Grossman 1986, Dyce et al. 2010). A exceção é nos suínos, que embora possuam a inserção do músculo trapézio na espinha da escápula como nas demais espécies, não possuem divisão evidente em partes cervical e torácica (Sisson \& Grossman 1986, Dyce et al. 2010).

Nos cães, o músculo omotransverso é superficial e recobre a borda craniodistal da escápula e se prolonga até a asa do atlas e processo transverso do áxis (Dyce et al. 2010). Nos ruminantes e suínos, o músculo encontra-se localizado na superfície lateral do pescoço, estendendo-se da asa do atlas até o ombro (Sisson \& Grossman 1986). Em equinos, a margem ventral funde-se com a parte cervical do músculo trapézio e une-se com o músculo cleidomastóideo (König \& Liebich 2011). Na paca por sua vez, esse músculo difere das observações encontradas na literatura para as espécies domésticas, pois o m. omotransverso é profundo e encoberto pelos músculos braquiocefálico e trapézio cervical.

Na camada profunda da musculatura do membro torácico, o músculo romboide também apresenta particularidades nos mamíferos domésticos e nos roedores. No geral, o $\mathrm{m}$. romboide encontra-se dividido em duas partes, cervical e torácica; entretanto, em carnívoros há uma terceira parte, chamada de parte capital que tem como origem a rafe tendinosa do pescoço (Cooper \& Schiller 1975, Greene 1955, Sisson \& Grossman 1986, Dyce et al. 2010, König \& Liebich 2011). Outra exceção entre os domésticos, que por sinal se assemelha aos achados da paca neste estudo, são os ruminantes, pois não possuem divisão definida entre as partes cervicais e torácicas do músculo romboide (Sisson \& Grossman 1986, Dyce et al. 2010, König \& Liebich 2011).

Nas pacas, não se observa divisão do músculo serrátil ventral, e sua inserção é na borda dorsal escápula. Nos animais domésticos, no rato e na cobaia por sua vez, o m. serrátil pode ser dividido em parte cervical e torácica; ambas se inserem no aspecto medial da escápula (Cooper \& Schiller 1975, Greene 1955, Dyce et al. 2010, König \& Liebich 2011).

O músculo peitoral superficial nas pacas origina-se da região esternal, enquanto a inserção ocorre na diáfise umeral craniolateral. Já nos animais domésticos, no rato e na cobaia, compõem-se por dois músculos, músculo peitoral transverso e músculo peitoral descendente. 0 primeiro emerge caudal ao músculo peitoral descendente desde o aspecto ventral do esterno e se mescla com a fáscia do antebraço. Enquanto que o segundo se origina do manúbrio esterno e se insere na crista do tubérculo maior do úmero (Cooper \& Schiller 1975, Dyce et al. 2010, König \& Liebich 2011).
Diferentemente dos animais domésticos, o músculo peitoral profundo nas pacas, não possui divisão visível e se insere no tubérculo maior do úmero. Os animais domésticos e a cobaia possuem o m. peitoral profundo dividido em cranial e caudal. Sendo que, a porção cranial se insere no músculo supraespinhoso, enquanto que a parte caudal se insere no tubérculo menor do úmero. A parte cranial é bem formada em equinos e suínos, e corresponde ao músculo subclávio de outros mamíferos domésticos (Cooper \& Schiller 1975, Dyce et al. 2010, König \& Liebich 2011). No rato, o $\mathrm{m}$. peitoral profundo possui ainda uma terceira porção ligado o processo xifoide do esterno ao ombro (Greene 1955).

0 músculo subescapular da paca assim como nos animais domésticos, no rato e na cobaia tem sua origem na fossa subescapular, enquanto que a inserção é no tubérculo menor do úmero (Greene 1955, Cooper \& Schiller 1975, Sisson \& Grossman 1986, König \& Liebich 2011).

No grupo caudal, o músculo deltoide, redondo maior e redondo menor também apresentam suas particularidades entre as espécies. 0 músculo deltoide apresenta-se indiviso em equinos e suínos, ou seja, apresentam uma cabeça que emergem desde a espinha da escápula através de uma aponeurose. Já nos ruminantes, nos carnívoros, no rato e na cobaia assim como observado na paca, apresentam duas divisões, acromial e escapular. Em todos os animais, a inserção ocorre na tuberosidade deltoide do úmero após passarem sobre o aspecto caudolateral da articulação do ombro (Greene 1955, Cooper \& Schiller 1975, Sisson \& Grossman 1986, König \& Liebich 2011).

Entre os mamíferos domésticos, o m. redondo menor é considerado um músculo redondo apenas em carnívoros; nos demais mamíferos domésticos é triangular (König \& Liebich 2011). 0 músculo redondo maior não é encontrado em suínos e é mais forte em gatos, pois ocorre fusão com o tendão do grande dorsal (Sisson \& Grossman 1986, Dyce et al. 2010). Na paca, o m. redondo menor é semelhante aos caninos, pois apresenta forma redonda e não se une ao grande dorsal.

No grupo extensor, o músculo tríceps apresenta três cabeças de origem (cabeça longa, cabeça lateral, cabeça medial) em equino, bovino, suíno e rato. Cão, caprino e a cobaia apresentam uma cabeça a mais, a cabeça acessória, que se origina da parte caudal do colo do úmero e se funde com as cabeças longa e lateral (Greene 1955, Cooper \& Schiller 1975, Sisson \& Grossman 1986, Dyce et al. 2010, König \& Liebich 2011). Na paca, o m. tríceps possui três cabeças, sendo que seus ventres são unidos e fortemente aderidos à diáfise umeral.

O músculo tensor da fáscia do antebraço difere em sua origem nos animais. Na paca, assim como nos carnívoros, no rato e na cobaia o m. tensor da fáscia lata possui uma ampla aponeurose do músculo grande dorsal; enquanto que em ruminantes e equinos, a origem desse músculo é na margem caudal da escápula (Cooper \& Schiller 1975, König \& Liebich 2011).

No grupo flexor, o músculo bíceps braquial em suínos, apresenta-se fusiforme e não é muito desenvolvido, diferente dos demais mamíferos domésticos e a cobaia escápula. Em gatos e no rato, o bíceps se insere apenas na tube- 
rosidade do rádio e nas cobaias se insere apenas na ulna; diferente do que ocorre no cão e na paca, pois como observado no estudo em questão, a inserção pode ocorrer tanto na tuberosidade ulnar quanto radial (Greene 1955, Cooper \& Schiller 1975, Sisson \& Grossman 1986, Dyce et al. 2010).

0 músculo coracobraquial, nas pacas, tem origem no processo coracoide da escápula e sua inserção por sua vez, ocorre na diáfise medial do úmero, semelhantemente aos animais domésticos, ao rato e à cobaia (Greene 1955, Cooper \& Schiller 1975, Sisson \& Grossman 1986, Schaller 1999, Dyce et al. 2010, König \& Liebich 2011).

Na paca, assim como nos carnívoros e nos ratos, o m. pronador redondo está presente de forma significativa (Greene 1955, König \& Liebich 2011). Nessas espécies, o $\mathrm{m}$. pronador redondo surge a partir do epicôndilo medial do úmero e converge no supinador, sendo que estes dois se inserem muito próximos. Enquanto que em ruminantes e suínos, este músculo não é visível; já no equino, é possível observar apenas uma pequena faixa assim como nas cobaias (Cooper \& Schiller 1975, Sisson \& Grossman 1986, Dyce et al. 2010, König \& Liebich 2011).

Os músculos do antebraço ora observados e identificados na paca: m. ancôneo; m. extensor radial do carpo; extensor obliquo do carpo; m. extensor digital comum; m. extensor digital lateral; $\mathrm{m}$. ulnar lateral; $\mathrm{m}$. flexor ulnar do carpo; $m$. flexor digital superficial; $m$. flexor digital profundo; $\mathrm{m}$. flexor radial do carpo e m. pronador redondo, se assemelham aos músculos dos animais domésticos, cobaia e rato (Greene 1955, Cooper \& Schiller, Sisson \& Grossman 1986, Dyce et al. 2010, König \& Liebich 2011) salvo algumas diferenças na origem e inserção de cada músculo e ausência em alguns animais.

De forma geral a origem e inserção de cada músculo que compõe o antebraço da paca são as mesmas observadas nas espécies domésticas que possuem cinco dedos e nos roedores (Greene 1955, Cooper \& Schiller 1975, Sisson \& Grossman 1986, Dyce et al. 2010, König \& Liebich 2011), todavia alguns músculos são distintos. 0 m. ancôneo em carnívoros e no rato tem sua origem no epicôndilo lateral do úmero (Greene 1955, Sisson \& Grossman 1986, König \& Liebich 2011). Enquanto que nos equinos, a sua origem é no terço distal da superfície caudal do úmero e em ruminantes, sua origem é na região caudal do terço distal do corpo do úmero e a borda óssea da fossa do olecrano. Nos suínos, o músculo ancôneo não está presente (Sisson \& Grossman 1986). Já na paca, a origem deste músculo é no epicôndilo medial assim como observado na cobaia (Cooper \& Schiller 1975).

\section{CONCLUSÃO}

Conclui-se que, salvo algumas variâncias, na origem e inserção de cada músculo e na fusão dos ventres de alguns grupos musculares, de forma geral, a musculatura do ombro, braço e antebraço de Cuniculus paca assemelham-se a dos animais domésticos e a de outros roedores.

Agradecimentos.- À Coordenação de Aperfeiçoamento de Pessoal de Nível Superior (CAPES), ao Conselho Nacional de Desenvolvimento Científico e Tecnológico (CNPq), à Fundação de Amparo a Pesquisa do Estado de São Paulo (Fapesp) e ao Programa de Pós-graduação em Cirurgia Veterinária da FCAV-Unesp pela colaboração com os projetos envolvidos nesse trabalho.

\section{REFERÊNCIAS}

Almeida Júnior G.A.D., Costa C., Monteiro A.L.G., Garcia C.A., Munari D.P. \& Neres M.A. 2004. Meat quality of lambs fed with high moisture corn silage in creep feeding. Revta Bras. Zootec. 33(4):1039-1047.

Aversi-Ferreira T.A., Mata J.R., Paula J.P. \& Silva M.S.L. 2006. Anatomia comparativa entre os músculos extensores do antebraço de Cebus libdinosus com humanos e outros primatas. Revta Eletrôn. Farmácia 3(2):13-15.

Bressan M.C., Oda S.H.I., Cardoso M.G., Miguel G.Z., Freitas R.T.F., Vieira J.O., Faria P.B., Savian T.V. \& Ferrão S.P.B. 2004. Composição de Ácidos Graxos dos Cortes Comerciais de Capivara (Hydrochaeris hydrochaeris, L. 1766). Ciênc. Agrotec. 28(6):1352-1359.

Schaller 0. 1999. Nomenclatura Anatômica Veterinária Ilustrada. Manole, São Paulo. 614p.

Cooper G. \& Schiller A.L. 1975. Anatomy of the Guinea Pig. Harvard University, Cambridge. $417 \mathrm{p}$.

Dubost G. \& Henry 0. 2006. Comparison of diets of the acouchy, agouti and paca, the three largest terrestrial rodents of French Guianan forests. J. Trop. Ecol. 22:641-651.

Dyce K.M., Wensing C.J.G. \& Sack W.O. 2010. Tratado de Anatomia Veterinária. $4^{\underline{a}}$ ed. Elsevier, Rio de Janeiro. 856p.

Eisenberg J.F. \& Redford K.H. 1999 Mammals of the neotropics: the central neotropics: Ecuador, Peru, Bolivia, Brazil. Vol.3. University of Chicago, Chicago. 609p.

Sisson S. \& Grossman J.D. 1986. Anatomia dos Animais Domésticos. 5a ed. Guanabara Koogan, Rio de Janeiro. 2048p.

Gomes C., Karam L.B. \& Macedo R.E.F. 2013. Atributos de qualidade da carne de paca (Agouti paca): perfil sensorial e força de cisalhamento [Quality atributes of paca meat (Agouti paca): sensory profile and shear force]. Arq. Bras. Med. Vet. Zootec. 65(2):559-565.

Greene E.C. 1955. Anatomy of the Rat. Hafner Publishing, New York. 370p.

König H.E. \& Liebich H.G. 2011. Anatomia dos Animais Domésticos: texto e atlas colorido. $4^{\mathrm{a}}$ ed. Artmed, Porto Alegre. 788p.

Lange R.R. \& Schmidt E.M.S. 2007. Rodentia: roedores silvestres (capivara, cutia, paca, ouriço), p.475-491. In: Cubas Z.S., Silva J.C.R. \& Catão-Dias J.A. (Eds), Tratado de Animais Selvagens: medicina veterinária. Roca, São Paulo. 2512p.

Mucciolo P. \& Paiva O.M. 1943. Cortes de carne suína, ovina e caprina em São Paulo: bases óssea e muscular dos diversos segmentos. Revta Fac. Med. Vet., São Paulo, 2(3):151-164.

Oliveira F.S. 2014. Assessing the effectiveness of 30\% sodium chloride aqueous solution for the preservation of fixed anatomical specimens: a 5-year follow-up study. J. Anat. 225(1):118-21.

Pachaly J.R., Acco A., Lange R.R., Nogueira T.M.R., Nogueira M.F. \& Ciffoni E.M.G. 2001. Order Rodentia (Rodents), p.225-237. In: Fowler M.E. \& Cubas Z.S. (Eds), Biology, Medicine, and Surgery of South American Wild Animals. State University Press, Iowa. 550p.

Redford K.H. \& Robinson J.G. 1991 Subsistence and commercial uses of wildlife in Latin America, p.6-23. In: Redford K.H. \& Robinson J.G. (Eds), Neotropical Wildlife Use and Conservation. University of Chicago Press, Chicago. 538p.

Rosa L.A., Silva F.O.C., Santos A.L.Q., Silva D.C.O. \& Lizardo F.B. 2012. Origem e distribuição do nervo axilar em tamanduá-bandeira (Myrmecophaga tridactyl). Biotemas 25(3):249-255.

Santos A.C., Bertassoli B.M., Oliveira V.C., Carvalho A.F., Rosa R.A. \& Manlaneres C.A.F. 2010a. Morfologia dos músculos do ombro, braço e antebraço do quati (Nasua nasua Linnaeus, 1758). Biotemas 23(3):165-173.

Santos A.C., Bertassoli B.M., Carvalho A.F., Rosa R.A. \& Manlaneres C.A.F. 2010b. Miologia comparada do membro torácico da mão-pelada (Procyon cancrivorus G. Cuvier, 1798). Revta Fac. Zootec. Vet. Agron. Uruguaiana 17(2):262-275.

Vavruk J.W. 2012. A importância do estudo da anatomia humana para o estudante da área de saúde. 0 Anatomista 2:4-35.

Vaz M.G.R., Lima A.R., Souza A.C.B., Pereira L.C. \& Branco E. 2011. Estudo morfológico dos músculos do antebraço de cachorro-do-mato-de-orelhas-curtas (Atelocynus microtis) e cachorro-do-mato (Cerdocyon thous). Biotemas 24(4):121-127. 